\title{
Pop-Up Windows and Information Retrieval
}

\author{
Stéphane Caro \\ INRIA Rhône-Alpes \\ 655, av. de l'Europe \\ 38330 Montbonnot Saint-Martin \\ FRANCE \\ Stephane.Caro@inrialpes.fr
}

\begin{abstract}
Paralinguistics organizers such as parenthesis, footnotes, and pop-up fields on screen, should play an important role signalling the writer's "minimization" intention when secondary points are concerned. This experiment investigated the role of pop-up fields compared to brackets in an information retrieval task. The results showed that, in this kind of task, putting secondary pieces of information into pop-up fields significantly speeded the search process compared to a condition in which the same information was displayed in brackets.
\end{abstract}

KEYWORDS Information Retrieval, Pop-up windows, Electronic Document Ergonomics

\section{INTRODUCTION}

A frequent task in a screen reading process is to find a piece of information in a set; either we are seeking information in a text (or a set of texts) in the hope of finding it, or we are seeking it knowing that it is in the content. In reading "from beginning to end", we admit that the reader does a categorization between main textual unit (TU) and secondary TU in order to modulate the depth of his processing. In a consultation for seeking information, the problem is to read the minimum of non-pertinent information before finding the pertinent. Categorization of TUs not only between main and secondary but also between various types of TUs could be crucial. We postulated that the use of popup windows could be a powerful means in order to facilitate the information retrieval task. If some TUs are hidden but correctly categorized, (with underlined words in the text for example) then the reader could either cheerfully ignore them, or explicitly seek the information in its locations; and in addition, such popup windowing of information decreases the amount of permanently displayed text and thus the text processing load.

\section{THE EXPERIMENT}

\subsection{Overview}

The objective of the experiment was to compare the effect of puting secondary TUs in pop-up windows with more classical para-linguistic organizers, like brackets, for a textual information retrieval task. Using the measurement of retrieval times for specific information (on main information TUs and secondary information TUs), we expected that the use of pop-up windows would decrease these information retrieval times.

\subsection{Material}

A small text database was constructed. The chosen texts present five cars. Each car description was five screen pages long. Two versions of this base were made: a «bracket» version in which the secondary TUs were at the same level as the main TUs but were between brackets (see Figure 1). In the «pop-up window» version, the same TUs were put in «pop-up windows». Underlined words (see Figure 1) enable the reader to: see the information in the pop-up with a mouse click, anticipate the type of information stored in the pop-up window.

Human-Computer Interaction: INTERACT'97 S. Howard, J. Hammond \& G. Lindgaard (editors)

Published by Chapman \& Hall OIFIP 1997 
Brackets

In the Bracket version the
complementary TU's were
displayed between brackets.
Alternatively, in the pop-up
window version (a pop-up
window is a small window
which appears in front of the
displayed text) there were one
or more underlined words
which activated a pop-up
window on a mouse click.

Figure 1: Brackets and pop-up window of the same text

\subsection{Subjects}

Twelve volunteers participated in the experiment. They were all native French speaking adults (average age 26 years) with an education level of at least bachelor +3 years.

\subsection{Retrieval Task}

Twelve questions had to be answered, six on each type of TU. The time taken to answer each question was measured.

\subsection{Experiment Plan}

Six subjects had pop-up windows and six had brackets. Three questions about main TUs and three questions about secondary TUs were destined to measure the evolution of response time at three points in the experiment (at the beginning, in the middle, at the end).

\subsection{Results}

An ANOVA with repeated measures was calculated on the question response time logarithm for each information type (main/secondary). Figure 2 shows the observed data and the interactions. For the search on the main information, the analysis shows that neither the presentation effect (brackets vs. pop-up) nor the moment effect was significant (at the beginning, in the middle, at the end). But their interaction was significant $[F$ $(2,20)=5.66 ; p<.02]$. Then, for the main information, if the presentation in a pop-up tends to give longer search times than the brackets presentation at the beginning, it is the contrary at the end of the session. For the search times on the secondary information TUs, the effect of the factors is not significant nor is their interaction.

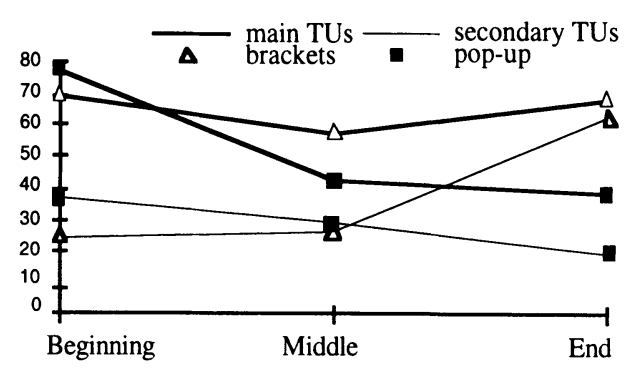

Figure 2: Response times (in sec.) at the 3 points

We considered the times needed for the main and secondary information TUs as two different measures and calculated a multivaried ANOVA. Globally, pop-up presentation gives significantly faster search times than brackets presentation [F- Wilks' Lambda $=4.6 ; p<.05$ ]. It is possible to make the same search decision in both types of information in the text: for the secondary information TUs $[\mathrm{F}(1,10)=5.1 ; \mathrm{p}<.05]$ and for the main information TUs $[\mathrm{F}(1,10)=7.2 ; \mathrm{p}<.03]$.

\subsection{Discussion}

For a retrieval task in a small textual base, the secondary information TUs distinction with pop-up has a facilitation effect in relation to a condition where the information is equally distinguished but simply with brackets. Indeed, even when the subjects were not familiar at the beginning with pop-ups, this experiment enabled us to observe an evolution of the relative efficiency of the two presentation forms. At the beginning, the pop-up windows tend to increase search times compared with brackets, but at the end of the experiment the tendency reverses. However, this evolution is only significant for searches for the main information TUs. This phenomenon seems to be due to a progressive improvement of the subject search times for the pop-up windows, but equally and curiously to a performance deterioration at the end of the session for the bracket subjects. It could be that the end session search test presented an a priori unsuspected difficulty compared to the searches at the beginning and the middle of the session. At the same time, the pop-up window group continue to improve their performances. However that may be, the final test showed that the pop-up window subjects are faster than brackets subjects and this is true for the searches on the main and the secondary TUs. 